\title{
Effectiveness of an Intervention Protocol to Improve Disordered Eating Behavior and Body Image in the Treatment of Body Image Disturbance and Eating Behaviors among Women
}

\author{
Sahar Badri Bageh Jan1, Seyedeh Zahra Alibakhshi ${ }^{2 *}$, Zahra Karami Baghtifuni ${ }^{2}$ \\ 1 MSc, Educational Psychology, Department of Psychology, Payame Noor University, Tehran, Iran \\ 2 Assistant Professor. Department of Psychology, Payame Noor University, Tehran, Iran
}

*Corresponding author: Seyedeh Zahra Alibakhshi, Educational Psychology, Department of Psychology, Payame Noor University, Tehran, Iran Tel: 09123453608

Email: z.alibakhshi@gmail.com
Received: 06 Mar. 2020 Accepted: 02 Jul. 2020 ePublished: 01 Nov. 2020

\begin{abstract}
Background and Objective: The manifestations of body image disorder in the eating disorder have been assessed on the cognitive-affective component; however, there is no evidence about these disorders on the behavior and perceptual components. Therefore, this study aimed to evaluate the effectiveness of an intervention protocol to improve unique eating behaviors and body image in the treatment of body image disorder and eating behaviors among women.

Materials and Methods: This quasi-experimental study was conducted based on a pretest-posttest design with a control group using an applied research method. The statistical population consisted of all women who referred to weight loss centers in Tehran, Iran, during 2018. The data were collected using the Dutch Eating Behaviors Questionnaire and Body Image Concern Inventory. In total, 30 women were selected through a random sampling method and assigned randomly into experimental $(n=15)$ and control ( $n=15)$ groups. Subsequently, the experimental group participated in eight intervention sessions per week intending to improve body posture and image.

Results: The results of the multivariate covariance analysis showed that the eating behavior $(\mathrm{P}=0.001, \mathrm{~F}=62.889)$ was statistically significant at 0.05 . The effect size of this intervention was approximately $70 \%$ on eating behavior and body image, which was acceptable in this study.

Conclusion: The intervention protocol to improve eating and body image had a significant effect on eating disorders and body image. However, there is a need for more replications, as well as more robust, randomized, and controlled trials to improve the methodological standard of intervention studies in this area.
\end{abstract}

Keywords: Body image, Eating behavior

\section{Background}

A wide range of abnormal eating behaviors with varying degrees of severity is involved in the eating disorder (ED), including fear of obesity, unhealthy weight control behaviors, and thoughts on food concerns. The EDs are at the end of the disordered eating spectrum, and these unhealthy behaviors do not warrant meeting diagnostic criteria [1].

The EDs usually begin in adulthood which is a stage of life associated with stressful events, such as leaving home for college [2]. Studies have shown that the prevalence of EDs among older students is $8 \%$ to $20.5 \%$ [3]. It is worrying that a significant proportion of college-aged students who show symptoms of ED have not been diagnosed and are not seeking treatment [4]. The results showed an intrinsic relationship of $\mathrm{ED}$ with body image disorder and perfectionism. In addition, the results of stepwise regression analysis revealed that body image disorder and perfectionism significantly predicted approximately $16 \%$ and $14 \%$ of the variance of EDs in female students, respectively [5]. Among patients diagnosed with EDs, body image disturbance is conceptualized to have a central role in the maintenance of psychopathology of ED [6]. For some individuals, weight and shape take on a level of importance that impairs functioning, notably individuals' evaluations of themselves. This importance along with the cognitive and behavioral efforts to control weight, shape, and eating behaviors, probably makes weight and shape become the focus of individuals' daily lives. Similarly, binge-eating disorder (BED) is a condition in which individuals experience a sense of loss of control while eating an unusually large amount of food [7]. One longitudinal study that tracked the psychopathology of the ED and ED 
behaviors weekly found that when overvaluation occurred during one week, it was followed by noncompensatory weight-control behaviors (e.g., strict dieting) the subsequent week [8].

Body image dissatisfaction is also forcefully correlated with and is often evaluated as a predicting and retaining factor of disordered eating [9]. Each person has two images of his/her body, one of which represents the status quo and the other signifies the ideal state of the body. Body image is the internal representation of one's outer appearance, which includes the physical, perceptual, and attitudinal dimensions of the individual [10], as well as mindfulness in the form of interventional measures to support EDs and body image [11]. Differences between the body image and the ideal image of the body can lead to varying degrees of distress or difficulty, probably followed by the development of a variety of mental disorders [12]. The ED is the third most common chronic disease among young people with increasing prevalence worldwide [13].

Weight gain and obesity in the world have been limited to adults for many years; however, they have also affected children and adolescents in the last two decades [14]. Impaired body image is one of the factors known to be effective in the development and maintenance of EDs. Research has shown that dissatisfaction with body image is associated with EDs. Due to the negative effects of body image on various functional areas of the individual, many therapists have proposed a plan to treat this disorder. Therefore, it seems that the evaluation of the structure of body image not only affects the functional aspects which take in to account one's perceptions of his/her body but also affects the way the body functions, including physical abilities [15].

Body image disorder and eating behaviors are associated with other indicators, such as self-esteem, anxiety, depression, and negative emotions longitudinally and transversely [16]. Biopsychosocial features of BED are associated with different forms of body image disturbance; however, no significant association has been observed between body image variables and body mass index. Moreover, BED frequency did not differ across body image variables [17]. In female adolescents, preoccupation showed the robust independent and mediating impacts on distress, dietary conservation, and binge eating; however, either the direct or indirect effects of dissatisfaction were significant on distress, overevaluation, and binge eating. On the other hand, direct and indirect effects of over-evaluation, dissatisfaction, and preoccupation were equal on distress and ED behaviors among adolescent males [18].

A study showed more dissatisfaction with body image and higher levels of distress in females [19]. A general population study of women reported the greater impact of over-evaluation and dissatisfaction in combination, compared to the effect of each of them in terms of association with psychological distress [19].

With this background in mind, since the evidence on the association among over-evaluation, dissatisfaction, and preoccupation is inconclusive, further research is required to elucidate the distinctiveness and clinical significance of these constructs. Furthermore, no a priori hypotheses were made on the relationships among image constructs.

\section{Objectives}

This study aimed to evaluate the effectiveness of an intervention protocol to improve unique eating behaviors and body image in the treatment of body image disorder and eating behaviors among females.

\section{Materials and Methods}

This quasi-experimental study was conducted based on a pretest-posttest design with a control group using an applied research method. The statistical population included all females with an ED and body image disorder who referred to weight loss centers in Tehran, Iran, during 2018. In total, 30 women with an ED and body image disorder were selected using the random sampling method and assigned randomly into experimental $(\mathrm{n}=15)$ and control $(n=15)$ groups. The inclusion criteria were female gender, as well as the diagnosis of ED and body image disorder. On the other hand, those who migrated and lacked proper cooperation, as well as the cases who did not meet favorable requirements (emergence of a specific disease during the study and implementation of dietary regimens concurrent with the study) were excluded from the study.

Subsequently, both groups were requested to complete the questionnaires before the intervention. The experimental group participated in eight twohour intervention sessions per week (8 weeks) that was conducted by the first author. It is worth mentioning that the intervention approach mainly focused to improve eating behavior and body image, and the posttest was administered to both groups at the end of the training sessions. Furthermore, the intervention protocol to "Improve Eating Behavior and Body Image" was based on the McNamara Eating Behavior and Body Image Improvement Package revised by Clearinghouse [1]. 
Regarding the ethical considerations, the participants were informed of the research objectives and procedures. Moreover, informed consent was obtained from them considering taking the tests and participating in the training sessions. It should be mentioned that they were assured of the anonymity and confidentiality of their information. The data were analyzed in SPSS software (version 21) through a multivariate analysis of covariance.

\section{Eating Behavior Questionnaire}

This self-administered scale was developed by Van Strien et al. with 33 items to measure an individual's eating style. It is rated on a 5-point Likert scale [20] $($ never $=1), \quad($ rarely $=2), \quad($ sometimes $=3), \quad($ often $=4)$, (very high $=5$ ) without lowest and highest scores. Moreover, it includes three independent scales of cognitive restraint $(\mathrm{n}=10)$, externality $(\mathrm{n}=10)$, and emotionality $(\mathrm{n}=13)$ [13]. The Eating Behavior Questionnaire consists of two sections, the first part of which seeks subjects' characteristics, such as height, weight, and presence or absence of periodic binge eating [20]. The second part of this scale consists of four items and three subscales to measure emotional, extrinsic, and inhibitory eating styles. Cronbach's alpha coefficient ranging from $77 \%$ to $83 \%$ revealed the good internal consistency of the Persian version of the Dutch Eating Behavior Questionnaire scale and subscales [21].

\section{Body Image Concern Inventory}

Body Image Concern Inventory (BICI) is a 19-item scale developed to assess the dysmorphic appearance concerns [22]. This self-report inventory is rated based on a 5-point Likert scale from never $=1$ to always $=5$. Cronbach's alpha coefficient of this scale was obtained at 0.93 in a college sample. This scale successfully distinguishes individuals with symptoms of body disorders and body malnutrition from those with bulimia or body deformity disorder determined by a clinical structural interview with a subclinical ED [22]. The highest and lowest scores of the not-reverse scored items were 95 and 19, respectively, with the high scores indicating a negative body image and low scores representing non-negative mental images of the individuals about themselves. Entezari and Alavizadeh [23] also reported internal consistency of $89 \%$ using Cronbach's alpha. Cronbach's alpha of this tool was determined at $87 \%$ in this study.

Table 1. Contents of the intervention protocol to improve eating behavior and body image

First Session
Introducing; Discussing diet repetition as a risk of overeating; Discussing the facts presented, reasons for dieting, history of diet, group
solidarity with persuasion; Discussing the dietary regimens and feelings about body weight by the participants; Assigning homework;

solidarity with persuasion; Discussing the dietary regimens and feelings about body weight by the participants; Assigning homework; Discussing the purpose of the daily eating behaviors and importance of doing it daily.

\section{Second Session}

Eating as a conflict: Changing eating behaviors; Discussing the main goals, logical basis of the program, weight, and calories specifically. Discussing changes in the rules of eating; Encouraging the individuals to exercise regularly; Presenting a list of several strategies that were appropriate to initiate a change in eating behavior with little attention to the realities of obesity and an inactive lifestyle.

\section{Third session}

Changing thought patterns: Providing leaflets to show the examples of positive and negative thinking about food, weight, dysfunctional thinking, and weekly memories of eating behavior; Explaining the circular relationship between thoughts, feelings, and behaviors shown in the pamphlet of changing patterns of thought; Exploring the types of dysfunctional thinking in the pamphlet of dysfunctional thinking that leads to dysfunctional behavior.

\section{Fourth Session}

Discussing self-esteem, perfectionism, and depression; Discussing positive traits and explaining the relationship among perfectionism, depression, and self-esteem; Discussing how to feed ourselves without food, self-esteem, and feedback exercises; Checking the assignments including stopping food control.

\section{Fifth Session}

Discussing anger and assertiveness; Focusing on positive emotions associated with self-feeding behavior; Encouraging the participants to continue focusing on changing their patterns of thinking and mixing time and activities for themselves throughout the day.

\section{Sixth Session}

Discussing cultural expectations about being slim, social pressure, and behavioral listing activities; Distributing photos that the participants wished to resemble and talking about it.

\section{Seventh Session}

Improving body image; Mirror training; Asking participants how they feel and focus on admiring their body with all its flaws and distortions in body image; Encouraging the participants to give each other honest feedback on the distortions they observe in real and ideal photos; Discussing some common cognitive distortions about body image and other features that women believed were related to their body image; Helping the participants to think more realistically about their body size; Checking the assignment.

\section{Eighth Session}

Presenting a summary; Presenting positive comments on any significant changes in the appearance of the group members; Examining what changes have occurred in their attractive behaviors; Reinforcing factors other than weight, attractiveness, and attention from others; Reminding the eighth session as the last session; Focusing on reviewing the progress of each woman and what she would do after leaving the intervention. 


\section{Results}

According to the results, the age range of the participants was 30-55 years, except for one case who was 45 years old. Regarding the marital and occupational status, all participants were married and employees in offices, respectively. As can be observed in Table 2, the mean scores of the body image (52.93) in the experimental group decreases after the intervention (38.06). Moreover, the mean scores of eating behaviors in the experimental group decreased from 103.86 to 72.26 after the intervention.

Table 5 showed that the eating behavior variable $(\mathrm{P}=0.001, \mathrm{~F}=62.889)$ was statistically significant at the level of 0.05 . Therefore, intervention protocol to improve eating behavior and body image had effects on the treatment of body image disorder and eating behaviors in the experimental group, compared to the control group who did not receive training. The effect sizes of this intervention on eating behavior and body image were estimated at $70 \%$ and $76 \%$, respectively.

Table 2. Mean $\pm S D$ of variables in the experimental and control groups at pretest-posttest

\begin{tabular}{lrcc}
\hline \multirow{2}{*}{ Variables } & \multicolumn{2}{c}{ Intervention Group } & Control Group \\
\cline { 2 - 4 } & \multicolumn{2}{c}{ Mean \pm SD } & Mean \pm SD \\
\hline Body & Pretest & $(52.93+9.12)$ & $(61.66+6.79)$ \\
image & Posttest & $(38.06+4.66)$ & $(62.53+6.93)$ \\
Eating & Pretest & $(103.86+8.83)$ & $101.66+9.49$ \\
behavior & Posttest & $(72.26+10.25)$ & $100.8+10.36$ \\
\hline
\end{tabular}

Table 3. Results of the Kolmogorov-Smirnov test showing the normality of pretest-posttest scores

\begin{tabular}{|c|c|c|c|c|}
\hline Variables & & & Z Smirnov Colmograph & Sig \\
\hline \multirow{2}{*}{ Body image } & Pretest & $(57.30+13.69)$ & 0.13 & 0.198 \\
\hline & Posttest & $(50.30+13.73)$ & 0.161 & 0.057 \\
\hline \multirow{2}{*}{ Eating behavior } & Pretest & $(102.76+9.08)$ & 0.133 & 0.185 \\
\hline & Posttest & $(86.53+17.69)$ & 0.106 & 0.200 \\
\hline
\end{tabular}

Table 4. Results of parallel regression lines

\begin{tabular}{lccccc}
\hline Source of change & Sum Squares & Df & Mean square & F & Sig (P) \\
\hline Body image posttest & 271.618 & 1 & 271.618 & 5.39 & 0.058 \\
Eating behavior posttest & 296.36 & 1 & 296.36 & 4.77 & 0.067 \\
\hline
\end{tabular}

Table 5. Covariance analysis of body image and eating behavior in the treatment group

\begin{tabular}{lcccccc}
\hline \multicolumn{1}{l}{ Groups } & Sum Squares & Df & Mean square & F & Sig (P) & Effect size \\
\hline Body image & 2651.608 & 1 & 2651.608 & 82.183 & 0.001 & 0.760 \\
Eating behavior & 4486.239 & 1 & 4486.239 & 62.897 & 0.001 & 0.708 \\
Body image & 838.885 & 26 & 32.265 & & & \\
Eating behavior & 1854.502 & 26 & 71.327 & & & \\
Body image & 81371.000 & 30 & & & & \\
Eating behavior & 233724,000 & 30 & & & & \\
Body image & 5468.300 & 29 & & & & \\
Eating behavior & 9083.467 & 29 & & & & \\
\hline
\end{tabular}

\section{Discussion}

The present study evaluated the effectiveness of an intervention protocol to improve eating behavior and body image in the treatment of body image disorder and eating behaviors in females referred to weight loss centers. The experimental group showed a significant decrease in ED and body image scores, compared to the control group. The results also revealed a difference between experimental and control groups in terms of ED and body image scores. Therefore, it can generally be concluded that the intervention protocol to improve eating behavior and body image can be effective in the treatment of patients with EDs and body image disorders.

The findings of this study are in line with the results of many previously conducted studies [24]. A systematic review of interventions on body image and eating disorders in middle-aged women revealed that seven interventions significantly improved body image scores at the posttest with significant improvements in ED using two of these interventions [24]. In addition to the significant improvement in body image, two interventions also reported significant changes in $\mathrm{ED}$ at the posttest [25]. These findings indicate the ability of interventions to improve body dissatisfaction and reduce EDs among middle-aged women. Interventions resulted in a slight to moderate improvement in body image, a slight reduction to the average idealized internalization of beauty, and a significant decrease in social comparison tendencies [26].

Previous studies have shown that dysfunctional body image is significantly persistent throughout adult life and is commonly reported in adolescent females with diet and ED [5]. Evidence from EDs at an early age suggests that unhealthy dietary behaviors occur in childhood and adolescence, which progress with individual development [27]. 
Negative attitudes and unrealistic expectations are especially prominent in body image disorders and can be influenced by gender, self-esteem, family, media, friends, and other social influences [27].

It can be concluded that several causes together have effects on body image disruption, as well as disordered eating behaviors and attitudes. It can be argued that industrialization, accelerated urbanization, improvements in the perception of esthetics and attitudes to obesity, prevalence of dietary regimes, and weight control equipment make adolescent and adult females more nervous and vulnerable to eating disorders [28].

Furthermore, there has been a strong emphasis on slimming, fear of obesity, as well as dissatisfaction with the shape and weight among women and adolescent females [29]. Studies have shown that dissatisfaction with body shape and weight is a good sign of EDs that caused feelings of anxiety, social isolation, and low self-esteem, as well as obsessivecompulsive thinking about weight loss. These thoughts can lead to increased anxiety and guilt, thereby resulting in the use of corrective behaviors, such as the consumption of laxatives to reduce anxiety. Most people with neurasthenia and anorexia have reported symptoms of anxiety before EDs [26-28].

One of the most important limitations of this study was the gathering of people at one time and one place. Other limitations included a lack of control over many of the moderating variables, including race, social class, and eating habits of other family members. Since this study was conducted only on females in Tehran, Iran, the results could not be generalized to other study settings, samples, or populations.

\section{Conclusions}

In this study, the females who received the intervention protocol were more self-accepting and less vulnerable to social pressures. The body does not have to be perfect to be admired and liked; however, for many women, the power is in being slim. Accordingly, they should be encouraged to abandon this inaccurate way of gaining power and focus on assertiveness and self-cultivation skills since a perfect body cannot guarantee happiness.

\section{Compliance with ethical guidelines}

Regarding the ethical considerations, written informed consent was obtained from the participants, and the study protocol was approved by the Ethics Committee of Payame Noor University, Tehran, Iran (IR.PNU.REC.1397.2532388).

\section{Acknowledgments}

The authors gratefully acknowledge all participants for their cooperation to conducting this study.

\section{Authors' contributions}

All authors contributed to developing this study.

\section{Funding/Support}

This study received no financial support for the research, authorship, and/or publication.

\section{Conflicts of Interest}

The authors stated that this study was conducted in the absence of any commercial or financial relationships that could be interpreted as a potential conflict of interest.

\section{References}

1. Khodabakhsh MR, Kiani F. Body image disturbance and perfectionism as predictor's factors of disordered eating behavior among female students. International Journal of Pediatrics. 2014; 2(4-3):399-406.

2. Hudson JI, Hiripi E, Pope HG Jr, Kessler RC. The prevalence and correlates of eating disorders in the National Comorbidity Survey Replication. Biological Psychiatry. 2007; 61(3):348-58. [DOI:10.1016/j.biopsych.2006.03.040] [PMID] [PMCID]

3. Tavolacci MP, Grigioni S, Richard L, Meyrignac G, Déchelotte P, Ladner J. Eating disorders and associated health risks among university students. Journal of Nutrition Education and Behavior. 2015; 47(5):412-20. [DOI:10.1016/ j.jneb.2015.06.009] [PMID]

4. Eisenberg D, Nicklett EJ, Roeder K, Kirz NE. Eating disorder symptoms among college students: prevalence, persistence, correlates, and treatment-seeking. Journal of American College Health. 2011; 59(8):700-7. [DOI:10.1080/ 07448481.2010.546461] [PMID] [PMCID]

5. Richard A, Rohrmann S, Lohse T, Eichholzer M. Is body weight dissatisfaction a predictor of depression independent of body mass index, sex and age? Results of a crosssectional study. BMC Public Health. 2016; 16(1):863. [DOI:10.1186/s12889-016-3497-8] [PMID] [PMCID]

6. Tabri N, Murray HB, Thomas JJ, Franko DL, Herzog DB, Eddy KT. Overvaluation of body shape/weight and engagement in non-compensatory weight-control behaviors in eating disorders: Is there a reciprocal relationship? Psychological Medicine. 2015; 45(14):2951-8. [DOI:10.1017/ S0033291715000896] [PMID]

7. American Psychiatric Association. Diagnostic and statistical manual of mental disorders. Arlington, VA: American Psychiatric Publishing; 2013.

8. Stice E, Gau JM, Rohde P, Shaw H. Risk factors that predict future onset of each DSM-5 eating disorder: predictive specificity in high-risk adolescent females. Journal of Abnormal Psychology. 2017; 126(1):38-51. [DOI:10.1037/ abn0000219] [PMID] [PMCID]

9. Cornelissen KK, McCarty K, Cornelissen PL, Tovée MJ. Body size estimation in women with anorexia nervosa and healthy controls using 3D avatars. Scientific Reports. 2017; 7(1):15773. [DOI:10.1038/s41598-017-15339-z] [PMID] [PMCID]

10. Sadibolova R, Ferrè ER, Linkenauger SA, Longo MR. Distortions of perceived volume and length of body parts. Cortex. 2019; 111:74-86. [DOI:10.1016/j.cortex.2018.10.016] [PMID]

11. Atkinson MJ, Wade TD. Does mindfulness have potential in eating disorders prevention? A preliminary controlled trial with young adult women. Early Intervention in Psychiatry. 2016; 10(3):234-45. [DOI:10.1111/eip.12160] [PMID]

12. Gaudio S, Brooks SJ, Riva G. Nonvisual multisensory impairment of body perception in anorexia nervosa: a systematic review of neuropsychological studies. PLoS One. 2014; 9(10):e110087. [DOI:10.1371/journal.pone.0110087] [PMID] [PMCID]

13. Irvine KR, McCarty K, McKenzie KJ, Pollet TV, Cornelissen KK, Tovée MJ, et al. Distorted body image influences body schema in individuals with negative bodily attitudes. 
Neuropsychologia. 2019; 122:38-50. [DOI:10.1016/j.neuro psychologia.2018.11.015] [PMID]

14. Yazdani N, Hosseini SV, Amini M, Sobhani Z, Sharif F, Khazraei $H$. Relationship between body image and psychological well-being in patients with morbid obesity. International Journal of Community Based Nursing and Midwifery . 2018; 6(2):175-84. [PMID] [PMCID]

15. Abbott BD, Barber BL. Embodied image: gender differences in functional and aesthetic body image among Australian adolescents. Body Image. 2010; 7(1):22-31. [DOI:10.1016/ j.bodyim.2009.10.004] [PMID]

16. Rohde P, Stice E, Marti CN. Development and predictive effects of eating disorder risk factors during adolescence: Implications for prevention efforts. The International Journal of Eating Disorders. 2015; 48(2):187-98. [DOI:10.1002/ eat.22270] [PMID] [PMCID]

17. Lydecker JA, White MA, Grilo CM. Form and formulation: examining the distinctiveness of body image constructs in treatment-seeking patients with binge-eating disorder. Journal of Consulting and Clinical Psychology. 2017; 85(11):1095-103. [DOI:10.1037/ccp0000258] [PMID] [PMCID]

18. Mitchison D, Hay P, Griffiths S, Murray SB, Bentley C, Gratwick-Sarll K, et al. Disentangling body image: the relative associations of overvaluation, dissatisfaction, and preoccupation with psychological distress and eating disorder behaviors in male and female adolescents. The International Journal of Eating Disorders. 2017; 50(2):11826. [DOI:10.1002/eat.22592] [PMID] [PMCID]

19. Mond JM, Hay PJ. Dissatisfaction versus over-evaluation in a general population sample of women. The International Journal of Eating Disorders. 2011; 44(8):721-6. [DOI:10.1002/ eat.20878] [PMID]

20. Van Strien T, Frijters JER, Bergers GPA, Defares PB. The Dutch Eating Behavior Questionnaire (DEBQ) for assessment of restrained, emotional, and external eating behavior. The International Journal of Eating Disorders. 1986; 5(2): 295-315. [DOI:10.1002/1098-108X(198602)5:2<295::AIDEAT2260050209>3.0.CO;2-T]

21. Nejati V, Alipour F, Saeidpour S, Bodaghi E. Psychometric properties of Persian version of Dutch eating behavior questionnaire. Journal of Fundamentals of Mental Health. 2018; 20(1):5-14.

22. Littleton HL, Axom DS, Pury CL. Development of the body image concern inventory. Behavior Research and Therapy. 2005; 43(2):229-41. [DOI:10.1016/j.brat.2003.12.006] [PMID]

23. Ghadakzadeh S, Ghazipour A, Khajeddin N, Karimian N, Borhani $M$. Body image concern inventory $(\mathrm{BICl})$ for identifying patients with BDD seeking rhinoplasty: using a Persian (Farsi) version. Aesthetic Plastic Surgery. 2011; 35(6):989-94. [DOI:10.1007/s00266-011-9718-8] [PMID]

24. Lewis-Smith H, Diedrichs PC, Rumsey N, Harcourt D. A systematic review of interventions on body image and disordered eating outcomes among women in midlife. The International Journal of Eating Disorders. 2016; 49(1):5-18. [DOI:10.1002/eat.22480] [PMID]

25. McLean SA, Paxton SJ, Wertheim EH. A body image and disordered eating intervention for women in midlife: a randomized controlled trial. Journal of Consulting and Clinical Psychology. 2011; 79(6):751-8. [DOI:10.1037/ a0026094] [PMID]

26. Alleva JM, Sheeran P, Webb TL, Martijn C, Miles E. A metaanalytic review of stand-alone interventions to improve body image. PLoS One. 2015; 10(9):e0139177. [DOI:10.1371/journal.pone.0139177] [PMID] [PMCID]

27. Grosick TL, Talbert-Johnson C, Myers MJ, Angelo R. Assessing the landscape: body image values and attitudes among middle school boys and girls. American Journal of Health Education. 2013; 44(1):41-52. [DOI:10.1080/ 19325037.2012.749682]

28. Jankauskiene R, Baceviciene M. Body image concerns and body weight overestimation do not promote healthy behaviour: evidence from adolescents in Lithuania. International Journal of Environmental Research and Public Health. 2019; 16(5):864. [DOI:10.3390/ijerph16050864] [PMID] [PMCID]

29. Springer SI, Levitt DH. Eating issues and body image in elementary school: detection and prevention strategies for school counselors. Journal of School Counseling. 2016; 14(2):n2. 